\title{
Cerebrospinal fluid shunt-induced chorea: case report and review of the literature on shunt-related movement disorders
}

\author{
Claudio M de Gusmäo, ${ }^{1}$ Aaron L Berkowitz, ${ }^{2}$ Albert Y Hung, ${ }^{2}$ \\ M Brandon Westover ${ }^{2}$
}

- Additional material is published online only. To view please visit the journal online (http://dx.doi.org/10.1136/ practneurol-2014-000913).

${ }^{1}$ Neurology Department, Boston Children's Hospital, Boston, Massachusetts, USA ${ }^{2}$ Neurology Department, Massachusetts General Hospital, Boston, Massachusetts, USA

\section{Correspondence to} Dr Claudio M de Gusmao, Neurology Department, Boston Children's Hospital, 300 Longwood Ave, Fegan 11, Boston, MA 02115, USA; claudio.degusmao@childrens. harvard.edu

Published Online First 4 July 2014
CrossMark

To cite: de Gusmäo CM, Berkowitz AL, Hung AY, et al. Pract Neurol 2015;15:42-44.

\section{INTRODUCTION}

Cerebrospinal fluid (CSF) diversion by shunting provides effective management of hydrocephalus. ${ }^{1}$ However, complication rates of CSF shunts range from 5\% to $50 \% .{ }^{1-3}$ Most common are shunt infections and mechanical failures; these may lead either to underdrainage (with re-emergence of hydrocephalus) or overdrainage (with intracranial hypotension and its potential complications, eg, subdural hematoma). ${ }^{3}{ }^{4}$ Misplacement and migration of shunt catheters may cause seizures, intracerebral haemorrhage, and/ or focal neurologic deficits, such as hemiparesis. ${ }^{12}$ We report a case of hemichorea after CSF shunt placement, and review the literature on CSF shunt-related movement disorders.

\section{CASE REPORT}

A 20-year-old woman with congenital hydrocephalus treated by CSF shunting presented with a purulent discharge from the site of a recent shunt revision and had a shunt infection. Her previous ventriculoperitoneal shunt was removed and a new ventriculopleural shunt was placed through a left occipital approach. There were no recent medication changes Five days later, she developed debilitating involuntary choreoathetotic movements of the right upper and lower limbs (see online supplementary video 1 ), interfering with feeding and walking. Her examination, apart from the involuntary movements, was normal, with no rigidity, tremor, weakness, or ataxia. Her serum electrolytes were normal. CT scan of head showed that the shunt catheter tip penetrated the ventricular wall into the brain parenchyma (figure 1); MR scan of brain showed that its tip abutted the posterior aspect of the putamen (figure 2). There was no worsening of hydrocephalus, stroke, or haemorrhage. The shunt was surgically repositioned. Her choreoathetosis started improving immediately
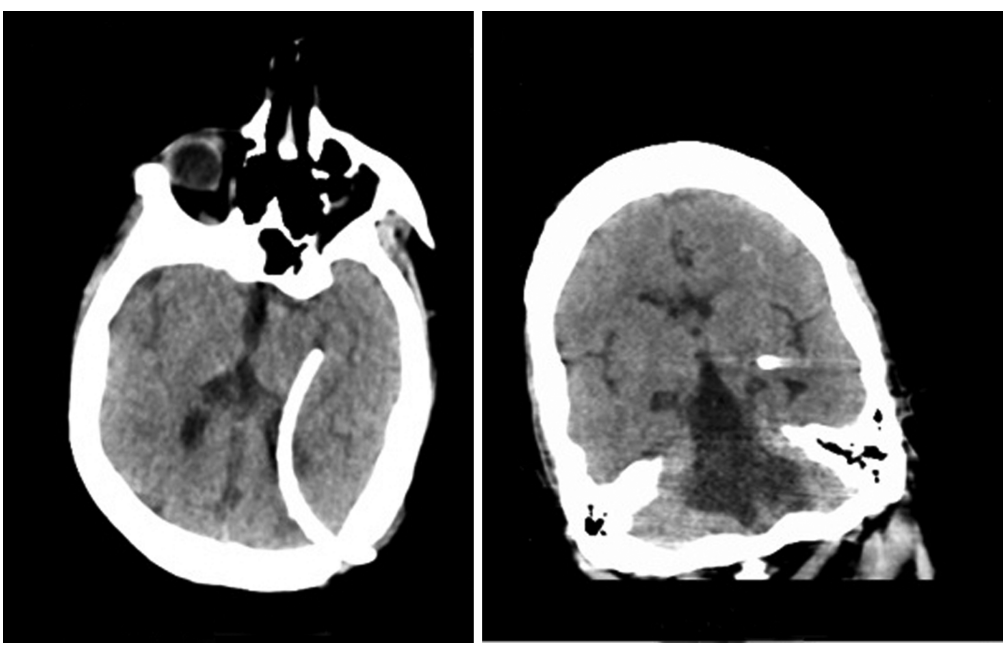

Figure 1 CT scan of head. Axial and coronal views demonstrating catheter in the brain parenchyma. 

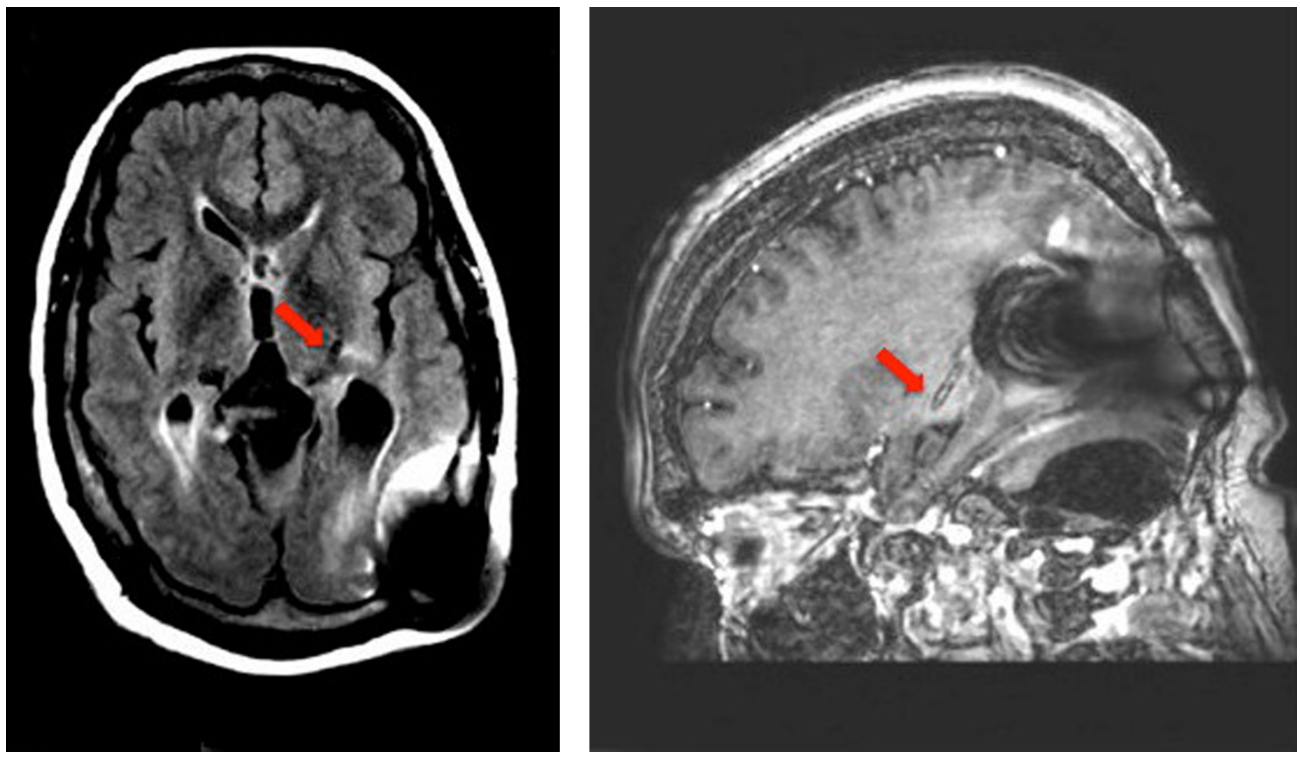

Figure 2 MR scan of brain. Axial (T2-FLAIR) and sagittal (T1 postcontrast) views show the cerebrospinal fluid shunt catheter tip terminating in the posterior putamen (arrows).

Table 1 Movement disorders complicating shunts

\begin{tabular}{|c|c|c|c|c|c|c|}
\hline Age & $\begin{array}{l}\text { Type of } \\
\text { movement }\end{array}$ & $\begin{array}{l}\text { Cause of } \\
\text { hydrocephalus }\end{array}$ & $\begin{array}{l}\text { Time after } \\
\text { shunting }\end{array}$ & Recovery and time & Mechanism & Reference \\
\hline 17 & Parkinsonism & Pineal gland germinona & $\begin{array}{l}\text { Not reported (but } \\
3 \text { days after roller } \\
\text { coaster ride) }\end{array}$ & Full, 1 month & $\begin{array}{l}\text { 'Shunt displacement with } \\
\text { hydrocephalus' }\end{array}$ & Lau et al ${ }^{11}$ \\
\hline 28 & Parkinsonism & $\begin{array}{l}\text { Ommaya reservoir for } \\
\text { methotrexate injection }\end{array}$ & 'Following treatment' & $\begin{array}{l}\text { 'Immediate after shunt } \\
\text { insertion' }\end{array}$ & Not reported & $\begin{array}{l}\text { Cheshire } \\
\text { et } a l^{16}\end{array}$ \\
\hline 32 & Parkinsonism & $\begin{array}{l}\text { 'Obstructive } \\
\text { hydrocephalus' }\end{array}$ & 3 months & Improved with levodopa & $\begin{array}{l}\text { 'Nigrostriatal pathway } \\
\text { concussion' }\end{array}$ & $\begin{array}{l}\text { Ochiai } \\
\text { et } a /^{13}\end{array}$ \\
\hline 59 & Parkinsonism & $\begin{array}{l}\text { 'Obstructive } \\
\text { hydrocephalus' }\end{array}$ & 3 months & Improved with levodopa & $\begin{array}{l}\text { 'Nigrostriatal pathway } \\
\text { concussion' }\end{array}$ & $\begin{array}{l}\text { Ochiai } \\
\text { et } a /^{13}\end{array}$ \\
\hline 38 & Parkinsonism & Neurocysticercosis & 3 days & Partial, 3 months & $\begin{array}{l}\text { 'Changes in intraventricular } \\
\text { pressure or malpositioning of } \\
\text { the shunt' }\end{array}$ & $\begin{array}{l}\text { Prashantha } \\
\text { et } a l^{6}\end{array}$ \\
\hline 46 & Parkinsonism & Aqueductal stenosis & 1 year & Partial with levodopa & $\begin{array}{l}\text { 'Parkinsonism associated } \\
\text { with V-P shunting' }\end{array}$ & $\begin{array}{l}\text { Sakurai } \\
\text { et } a l^{17}\end{array}$ \\
\hline 64 & Parkinsonism & Aqueductal stenosis & 4 months & None & 'Slit-ventricle syndrome' & Yomo et $a l^{18}$ \\
\hline 26 & Parkinsonism & Encephalitis & 7 months & Full, 2 months & 'Shunt malfunction' & $\begin{array}{l}\text { Tokunaga } \\
\text { et } a l^{12}\end{array}$ \\
\hline 11 & $\begin{array}{l}\text { Akinesia/ } \\
\text { parkinsonism }\end{array}$ & Tectal glioma & 'Days' & $\begin{array}{l}2 \text { weeks, Partial } \\
\text { improvement with } \\
\text { bromocriptine and } \\
\text { levodopa }\end{array}$ & $\begin{array}{l}\text { 'Recurrent hydrocephalus and } \\
\text { tectal glioma' }\end{array}$ & Rebai et $a l^{19}$ \\
\hline 32 & $\begin{array}{l}\text { Tremor/ } \\
\text { parinaud's } \\
\text { syndrome }\end{array}$ & Neurocysticercosis & Not reported & $\begin{array}{l}\text { 'Several days after shunt } \\
\text { revision' }\end{array}$ & $\begin{array}{l}\text { Hydrocephalus or direct } \\
\text { trauma to basal ganglia }\end{array}$ & Keane $^{7}$ \\
\hline 68 & Ballism & $\begin{array}{l}\text { Normal pressure } \\
\text { hydrocephalus }\end{array}$ & $\begin{array}{l}\text { 'Upon awakening } \\
\text { from surgery' }\end{array}$ & Not reported & Not reported & $\begin{array}{l}\text { Walker } \\
\text { et } a l^{20}\end{array}$ \\
\hline 14 & Torticollis & Aqueductal stenosis & 12 years & Full, 1 year & 'Local in the neck' & Singh et $\left.a\right|^{21}$ \\
\hline 14 & $\begin{array}{l}\text { Ataxia } \\
\text { cerebrospinal } \\
\text { fluid deficits }\end{array}$ & Congenital Chiari I & 13 years & 'Near complete', 1 week & $\begin{array}{l}\text { 'Positive rostral pressure } \\
\text { gradient through stenotic } \\
\text { foramen magnum' }\end{array}$ & Elliot et $\left.a\right|^{22}$ \\
\hline 24 & Hemichorea & Meningitis & 2 weeks & Complete, 'few days' & Not reported & $\begin{array}{l}\text { Alakandy } \\
\text { et } a l^{5}\end{array}$ \\
\hline
\end{tabular}


postoperatively, and had largely resolved spontaneously at 2 weeks when she was discharged to rehabilitation (see online supplementary video 2 )

\section{DISCUSSION}

Movement disorders related to cerebrospinal fluid (CSF) shunting are rare, though parkinsonism, ataxia, ballism and torticollis may occasionally occur (table 1). We could find only one previous report of shunt-related hemichorea, which, as here, resolved after shunt repositioning. ${ }^{5}$

The mechanism of ventriculoperitoneal (VP) shuntrelated movement disorders has been attributed to 'altered CSF dynamics', ${ }^{6}$ whereby shunt obstruction causes acute hydrocephalus, with shearing, torsion, or ischaemia of striatal projections. ${ }^{7-14}$ Although there is a report of chorea accompanying acute hydrocephalus and resolving after shunting, in that case the movements were generalised and presumed secondary to caudate head compression by the dilated lateral ventricle. ${ }^{15}$ In our case, the movement disorder probably resulted from direct striatal irritation by the shunt catheter itself, as the movement disorder emerged in the setting of normal shunt function with no radiological evidence of worsening hydrocephalus, and promptly resolved after shunt repositioning.

\section{CONCLUSION}

Hemichorea is an unusual complication of CSF shunt placement. Clinicians should be attentive to focal deficits including unilateral movement disorders in patients with CSF shunts, as prompt surgical intervention may alleviate debilitating neurological symptoms and minimise the risk of long-term complications.

Contributors CMdG: original idea, drafted the manuscript, edited images, performed literature review. ALB: original idea, revised the original manuscript and images, critical appraisal of literature review. AYH and MBW: critical review and modifications of the final manuscript; appraisal of literature review and associated media.

\section{Competing interests None.}

Patient consent Obtained.

Provenance and peer review Not commissioned. Externally peer reviewed. This paper was reviewed by Tony

Amato-Watkins, Cardiff, UK.

\section{REFERENCES}

1 Hdeib A, Cohen AR. Hydrocephalus in children and adults. In: Ellenbogen RG, Abdulraf SI, Sekhar LN, eds. Principles of neurological surgery. Philadelphia, PA: Elsevier, 2012:105-27.

2 Aschoff A, Kremer P, Hashemi B, et al. The scientific history of hydrocephalus and its treatment. Neurosurg Rev

1999;22:67-93; discussion 94-5. http://www.ncbi.nlm.nih.gov/ pubmed/10547004

3 Browd SR, Ragel BT, Gottfried ON, et al. Failure of cerebrospinal fluid shunts: part I: obstruction and mechanical failure. Pediatr Neurol 2006;34:83-92.

4 Browd SR, Gottfried ON, Ragel BT, et al. Failure of cerebrospinal fluid shunts: part II: overdrainage, loculation, and abdominal complications. Pediatr Neurol 2006;34:171-6.
5 Alakandy LM, Iyer RV, Golash A. Hemichorea, an unusual complication of ventriculoperitoneal shunt. J Clin Neurosci 2008;15:599-601.

6 Prashantha DK, Netravathi M, Ravishankar S, et al. Reversible parkinsonism following ventriculoperitoneal shunt in a patient with obstructive hydrocephalus secondary to intraventricular neurocysticercosis. Clin Neurol Neurosurg 2008;110:718-21.

7 Keane JR. Tremor as the result of shunt obstruction: four patients with cysticercosis and secondary parkinsonism: report of four cases. Neurosurgery 1995;37:520-2. http://www.ncbi. nlm.nih.gov/pubmed/7501120 (accessed 9 Jan 2014).

8 Halterman MW, Vates GE, Riggs G. Teaching NeuroImage: tremor in aqueductal stenosis and response to endoscopic third ventriculostomy. Neurology 2007;68:E29-31.

9 Jellinek EH. Not Parkinson's disease: neurologists' mistakes with a diversion into adult hydrocephalus. Pract Neurol 2008;8:322-4.

10 Krauss JK, Regel JP, Droste DW, et al. Movement disorders in adult hydrocephalus. Mov Disord 1997;12:53-60.

11 Lau C-I, Wang H-C, Tsai M-D, et al. Acute Parkinsonian syndrome after ventriculoperitoneal shunt malfunction caused by a roller coaster ride. Clin Neurol Neurosurg 2011;113:423-5.

12 Tokunaga $\mathrm{H}$, Shigeto $\mathrm{H}$, Inamura $\mathrm{T}$, et al. A case of severe parkinsonism induced by failure of ventriculo-peritoneal shunt for aqueductal stenosis. Rinsho Shinkeigaku 2003;43:427-30. http://www.ncbi.nlm.nih.gov/pubmed/14582370 (accessed 9 Jan 2014).

13 Ochiai H, Yamakawa Y, Miyata S, et al. L-dopa effective parkinsonism appeared after shunt revision of the aqueductal stenosis: report of two cases. No To Shinkei 2000;52:425-9. http://www.ncbi.nlm.nih.gov/pubmed/10845212 (accessed 13 Jan 2014).

14 Akiyama TT, Tanizaki Y, Akaji K, et al. Severe parkinsonism following endoscopic third ventriculostomy for noncommunicating hydrocephalus-case report. Neurol Med Chir (Tokyo) 2011;51:60-3. http://www.ncbi.nlm.nih.gov/pubmed/ 21273748 (accessed 13 Jan 2014).

15 Voermans NC, Schutte PJ, Bloem BR. Hydrocephalus induced chorea. J Neurol Neurosurg Psychiatry 2007;78:1284-5.

16 Cheshire WP, Ehle AL. Hemiparkinsonism as a complication of an Ommaya reservoir. Case report. J Neurosurg 1990;73:774-6.

17 Sakurai T, Kimura A, Yamada M, et al. Rapidly progressive parkinsonism that developed one year after ventriculoperitoneal shunting for idiopathic aqueductal stenosis: a case report. Brain Nerve 2010;62:527-31. http://www.ncbi.nlm.nih.gov/pubmed/ 20450100 (accessed 26 Jan 2014).

18 Yomo S, Hongo K, Kuroyanagi T, et al. Parkinsonism and midbrain dysfunction after shunt placement for obstructive hydrocephalus. J Clin Neurosci 2006;13:373-8.

19 Rebai RM, Houissa S, Mustapha ME, et al. Akinetic mutism and parkinsonism after multiple shunt failure: case report and literature review. J Neurol Surg A Cent Eur Neurosurg 2012;73:341-6.

20 Walker FO, Hunt VP. Ballism: an association with ventriculoperitoneal shunting. Neurology 1990;40:1004. http://www. ncbi.nlm.nih.gov/pubmed/2345602 (accessed 9 Jan 2014).

21 Singh G, Kaif M, Ojha BK, et al. Torticollis as a late complication of ventriculoperitoneal shunt surgery. J Clin Neurosci 2011;18:865-6.

22 Elliott R, Kalhorn S, Pacione D, et al. Shunt malfunction causing acute neurological deterioration in 2 patients with previously asymptomatic Chiari malformation Type I. Report of two cases. J Neurosurg Pediatr 2009;4:170-5. 